\title{
The CUSP/CORE problem from a 2D view
}

\author{
Juan Carlos Basto-Pineda*, Claudia Mendes de Oliveira, Carlos Eduardo Barbosa \\ Instituto de Astronomia, Geofísica e Ciências Atmosféricas, Universidade de São Paulo \\ Rua do Matão 1226, São Paulo SP, Brazil \\ E-mail: basto-pineda@usp.br, oliveiraeastro.iag.usp.br, \\ carlos.barbosa@usp.br
}

\section{Philippe Amram, Valentin Perret}

Aix Marseille Université, CNRS, LAM (Laboratoire d'Astrophysique de Marseille)

UMR 7326, 13388, Marseille, France

E-mail: philippe.amrameoamp.fr, valentin.perreteoamp.fr

\begin{abstract}
It has been claimed for a long time that the shapes of galactic dark matter haloes in numerical simulations are not consistent with those of real galaxies, as inferred from observations. While the former may have a steep density profiles in the center, the latter may reveal nearly flat central dark matter distributions, and that is why this controversy is known as the "cusp/core problem". Nevertheless, it is important to highlight that most of the comparisons have been made using results from dark-matter-only simulations, e.g, ignoring the real effects of baryonic feedback at galactic scales. On the other hand, many observational works rely on the assumption that galactic disks are highly symmetric rotating structures, which is rarely the case either for light distributions or for velocity fields.

In this work we use high-resolution $\mathrm{H} \alpha$ velocity fields to make a comparison between the results obtained when perfectly rotating disks are assumed, against the results obtained using a harmonic decomposition to effectively separate non-circular motions from the pure circular component, which may be a better tracer of the underlying potential. We have selected a sample of galaxies analyzed and published by different authors. These have been considered good candidates for mass modeling, and have, therefore, played a role into the cusp/core controversy.

Following a classical approach to create mass models of rotating galaxies we show that conclusions about the cuspiness of the DM halo could be affected by the way the data are treated, and that there is a general trend towards higher mass-to-light ratios of baryonic components when the "pure rotation" assumption is considered.
\end{abstract}

VIII International Workshop on the Dark Side of the Universe

June 10-15, 2012

Búzios, Rio de Janeiro, Brasil

\footnotetext{
* Speaker.
} 


\section{Introduction}

It has been claimed for a long time that there is a mismatch between the shape of dark matter galactic haloes predicted by $\Lambda \mathrm{CDM}$ cosmological simulations of structure formation, and the shape inferred from observations of late type rotating spiral galaxies. The former approach suggests a density profile becoming extremely sharp towards the center (cusp) (e.g Dubinski \& Carlberg 1991; Navarro et al. 1996b, 1997), while the latter indicates a flat density profile (core) in the central region (e.g. Athanassoula et al. 1987; e.g., Begeman et al. 1991). This apparent contradiction is known as the cusp/core problem and it is considered as one of the greatest challenges for current numerical cosmology (for an up-to-date review see de Blok, 2010).

Nevertheless, some important facts should be noticed before considering this contradiction a problem for the $\Lambda \mathrm{CDM}$ cosmological paradigm. First, we should keep in mind that this problem was noticed using dark-matter-only numerical simulations; it means that the effect of baryons during the galaxy assembly process was completely neglected. Today it is widely accepted that in some cases baryonic feedback in the form of star formation, supernovae explosions, and galactic winds, for instance, could play a significant role in modeling the potential well and density profiles of the different components of a galactic system (e.g. Navarro et al. 1996a), even transforming cusps into cores (Governato 2012).

On the other hand, the analysis of observational data has some inherent uncertainties which are not always properly taken into account. For example, most of the works fitting rotation curves of disk galaxies implicitly consider that late-type spiral galaxies are fully-rotating systems, even though many of these systems exhibit non-negligible random motions. Most typical approaches assume axial symmetry as well, although this is not the case in general, spiral arms and bars being the most evident counter-examples. Thus, it is possible to say that the cusp/core controversy has not been definitively solved yet, and that there are still several facts which could be influencing the possible scenarios.

In this paper we revisit the cusp/core controversy using the galaxies and data from the GHASP survey. The main focus is the comparison between the results obtained when velocity fields are treated in two different ways: considering that all velocities are circular, or disentangling noncircular motions via a harmonic decomposition.

\section{Mass models of galaxies}

In order to derive the shape and concentration of dark matter haloes of real spiral galaxies, the typical approach is to assume that rotation curves/velocity fields are fair tracers of the underlying potential, so that $v_{c}(r)=\sqrt{-\nabla \phi \cdot r}$ holds. This method implicitly assumes that deviations from axial symmetry in mass and velocity distributions are low, and that the whole system is in a state of dynamical equilibrium. Then one creates a mass model of the galaxy using building blocks representing the real observed components (a stellar bulge, a stellar disk, or a gas disk, for instance, as given by the photometry), and possibly a model for the unseen dark matter halo. Given the linearity of the gravitational potential and the gradient operator, then it follows that velocity components can be added in quadrature $v_{c}^{2}(r)=V_{\text {Disk }}^{2}+V_{\text {Bulge }}^{2}+V_{\text {Gas }}^{2}+V_{\text {Halo }}^{2}+V_{\ldots}^{2} \cdots$. 


\begin{tabular}{ccccccccc}
\hline \hline name & $\begin{array}{c}\text { D } \\
\text { Mpc }\end{array}$ & $\begin{array}{c}\text { Spatial Resol. } \\
\text { pc }\end{array}$ & $\begin{array}{c}\text { Incl. } \\
\text { deg }\end{array}$ & $\begin{array}{c}\text { P.A } \\
\text { deg }\end{array}$ & $\begin{array}{c}\text { Vsys } \\
\mathrm{km} \mathrm{s}^{-1}\end{array}$ & Type & Ref. \\
\hline ugc3876 & - & 14.5 & $\sim 239$ & $59 \pm 5$ & $178 \pm 3$ & $854 \pm 2$ & SA(s)d & Sp08 \\
\hline ugc5842 & ngc3346 & 15.2 & $\sim 258$ & $47 \pm 9$ & $112 \pm 2$ & $1245 \pm 1$ & SB(rs)cd & Sp08 \\
\hline ugc7045 & ngc4026 & 11.4 & $\sim 116$ & $68 \pm 2$ & $99 \pm 2$ & $758 \pm 1$ & SA(s)c & Sp08 \\
\hline ugc7876 & ngc4365 & 14.5 & $\sim 211$ & $53 \pm 9$ & $944 \pm 1$ & $164 \pm 3$ & SAB(s)d & Sp08 \\
\hline ugc8334 & ngc5055 & 9.8 & $\sim 109$ & $66 \pm 1$ & $100 \pm 1$ & $484 \pm 1$ & Sbc & dB08 \\
\hline ugc9866 & ngc5949 & 7.4 & $\sim 248$ & $56 \pm 6$ & $148 \pm 2$ & $430 \pm 1$ & SBc & dP12/S05 \\
\hline ugc11300 & ngc6689 & 8.4 & $\sim 151$ & $70 \pm 3$ & $168 \pm 2$ & $482 \pm 1$ & SABc & dP12/S05 \\
\hline \hline
\end{tabular}

Table 1: Sample information. Spatial resolution is measured from the average psf of the observatory. The last column lists at least one article where the galaxy was published in the context of the cusp/core problem. References: Sp08 = Spano et al. 2008, dB08 = de Blok et al. 2008, dP12 = del Popolo 2012, S05 = Simon et al. 2005.

\section{Observations and data reduction}

\subsection{The GHASP Survey}

The data used in this work comes from the GHASP (Gassendi $\mathrm{H} \alpha$ survey of SPirals) project, a compilation of $\mathrm{H} \alpha$ data cubes obtained with a scanning Fabry-Perot mounted on the $1.93 \mathrm{~m}$ telescope at the Observatoire de Haute-Provence, for a large sample of nearby spiral and irregular galaxies (http://fabryperot.oamp.fr/PerotFabry/). From these data cubes, the GHASP team has derived $\mathrm{H} \alpha$ maps, velocity fields, position-velocity diagrams, rotation curves and kinematical parameters as reported in (Epinat et al. 2008). The accuracy is about $5 \mathrm{~km} \mathrm{~s}^{-1}$ on average in velocity and 2 arcsec in spatial resolution (limited by seeing) (Garrido et al. 2003).

Additionally, images in the Rc band were obtained for a sub-sample of galaxies including those analyzed here, using a $1.2 \mathrm{~m}$ telescope at the same observatory between 2002 and 2009. Data are described elsewhere (Barbosa et al. 2013, in preparation).

\subsection{Sample selection}

For this comparison we have chosen a sample of 7 spiral galaxies published by other authors in the context of the cusp/core problem (i.e. considered as suitable for mass modeling procedure). These galaxies are close enough as to allow probing the first inner kiloparsec of the rotation curve, where the cusp/core discrepancy is more severe. Additionally, we require that the galaxies have moderate inclinations $70^{\circ}>i>30^{\circ}$ and high-quality velocity maps. The source for the distance measurement to each galaxy and morphological type can be found in Epinat et al. (2008). The geometrical parameters are those determined from the kinematics by the GHASP team and reported in the same paper. Basic information is summarized in table 1.

\subsection{Stellar Rotation Curves}

Images in the Rc band of our galaxies were used in order to determine the stellar contributions to the rotation curve. After applying the instrumental zero point calibration, sky subtraction, and galactic extinction corrections, surface brightness profiles were drawn using the task ELLIPSE within the IRAF package. Then we run a typical decomposition of the profiles into a Sérsic bulge and an exponential disk, even though our images suggest the existence of more or different components in some cases. We insist in doing it in this way for simplicity, and because we are just 
interested in the impact on the results owed to the way the velocity field is treated. Thus, some of the bulge/disk decompositions presented here could be a poor physical description, fact that will be further explored in a forthcoming paper using $2 \mathrm{D}$ image decompositions instead of 1D surface brightness profiles.

Once we had the bulge/disk decompositions we created generic rotation curves for each component assuming mass-to-light ratios $\gamma$ equal to one (in $M_{\odot} / L_{\odot}$ ), assuming spherical symmetry for

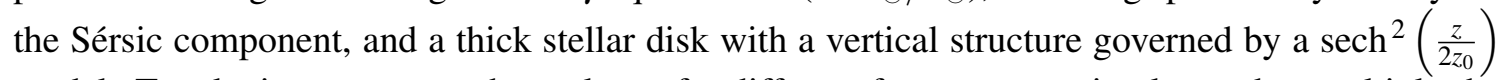
model. To take into account other values of $\gamma$ different from one we simply need to multiply the velocity amplitudes by $\sqrt{\gamma}$.

\section{Methodology}

\subsection{From Velocity Maps to Rotation Curves}

In the past, rotation curves were built using long-slit spectroscopy along the major axis. Even though these points have the lowest velocity uncertainties, the lack of two-dimensional information rules out the possibility of measuring and correcting for non-circular motions. Additionally, slits used to be placed along the photometric major axis which does not necessarily match the kinematical one. For these reasons, several authors now use high resolution velocity fields (e.g. Spano et al. 2008; Kuzio de Naray et al. 2009), which is also the case for this work.

Here we propose to build the final rotation curve in two different ways. The first one consists in assuming perfect circular velocities in a thin disk, so the inferred circular velocity and the lineof-sight measured component would be simply related by

$$
v_{\text {los }}(r)=v_{\text {sys }}+v_{c}(r) \frac{\sin (i) \cos (\Psi)}{\sqrt{\cos ^{2}(\psi)+\frac{\sin ^{2}(\psi)}{\cos ^{2}(i)}}}
$$

where $v_{\text {los }}$ represents line-of-sight velocity component, $v_{c}$ the interpreted circular velocity, $v_{s y s}$ is the systemic velocity of the galaxy as a whole, $i$ holds for inclination and $\Psi$ is the polar angle from the major axis in the plane of the sky. This method will be referred everywhere in this work as the "pure rotation" assumption, and could be considered as the natural extension of the long-slit spectroscopy technique, except for the fact that now we include in the fit a much higher number of velocity points and misalignment uncertainties disappear. We have defined the final uncertainty as

$$
\sigma_{V r o t}=\frac{\sigma_{V l o s}}{\cos (\theta) \sin (i)}
$$

where $\theta$ holds for the polar angle in the plane of the disk. We have excluded an angular region of $22.5^{\circ}$ around the minor axis due to large errors when $\theta$ is large. We end up not with a rotation curve but a cloud of velocity points as those showed in blue in Figure 1 for ugc8334. We avoided to bin this cloud because this would introduce an uncertainty in the radius, and it is not necessary for the fitting procedure.

On the other hand, we will define our alternative rotation curve by means of a harmonic decomposition of the whole velocity field based on the Kinemetry package by Krajnović (2006), which allows us to separate non-circular motions from the pure rotating component using

$$
V_{\text {los }}=a_{0}+\sum_{i} a_{i} \sin (i \psi)+b_{i} \cos (i \psi)
$$




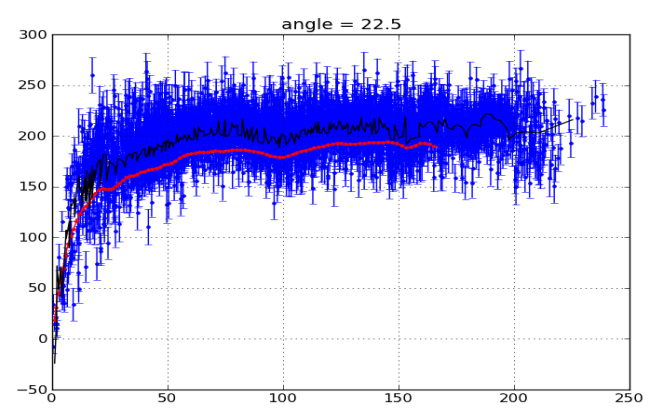

Figure 1: Kinemetry rotation curve for ugc8334 (red). "Pure rotation" velocity cloud (blue) and its binned version (black). Velocities are in $\mathrm{km} \mathrm{s}^{-1}$.

In this equation, the $a_{0}$ coefficient will be related to $V_{\text {sys }}$ and to coherent motions out of the galactic plane, $b_{1}$ to the circular velocity, $a_{1}$ to the coherent expansion velocity, and all other coefficients will absorb the effect of random motions.

In this case we will define the uncertainty in a different way, adding in quadrature the statistical uncertainty from the kinemetry fitting procedure and the systematic uncertainty due to the uncertainty in the geometrical parameters, because it seems that the statistical uncertainties alone are unrealistically low as pointed out by Simon et al. (2005). In fact, they are much lower than the uncertainties defined for the "pure rotation" velocity cloud, so this is an attempt to make them comparable. Nonetheless, it should be noted that reduced $\chi^{2}$, s found later on for both rotation curves can not be compared directly in a statistical sense, given that uncertainties have been defined in these different ways.

To calculate the systematic uncertainty we follow Simon et al. (2005), drawing a Gaussian probability distribution function for each geometrical parameter, picking 1000 combinations and drawing the resulting 1000 rotation curves. Then we take their mean as the final RC and their standard deviation as the systematic uncertainty. The final kinemetry rotation curve for our canonical example is shown in red in Figure 1. The black line represents a binned version of the blue velocity cloud, just to give an impression of its global behavior. Note that the black line is systematically offset to higher velocities than the red one, which is expected from the fact that in the former case we are overestimating the real pure circular velocity component.

\subsection{Rotation Curves Fitting and DM Halo Density Profiles}

We will just consider the stellar disks and bulges described above, and two different models for the dark matter halo: a Navarro-Frenk-White profile (NFW) and an Isothermal Sphere model (ISO). The first one is a good description of dark matter haloes in numerical simulations (Navarro, 1996b), and it grows as steep as $\rho \propto r^{-1}$ towards the center. The second is flat in the central region $\left(\rho \propto r^{0}\right)$ and has been successfully used in the literature to fit real data (e.g. Athanassoula et al. 1987). Both of them are characterized by two parameters, a central density $\rho_{0}$ and a characteristic radius $R_{0}$.

We run two very simple tests. The first one is known as the zero disk fit, and consists in fitting the rotation curve using the DM halo alone, i.e. neglecting the contribution of stellar components, therefore giving an upper limit on the concentration of the halo density. 
The second test is known as the maximal disk fitting, and consists in allowing the maximum contribution of baryons to the rotation curve before fitting the mass model, in order to put a lower limit on the concentration of the DM halo. In our case we have included a bulge component, so we call this approach the maximal stars fitting instead of maximal disk. To make the following procedures comparable for the kinemetry RC and the "pure rotation" velocity cloud, the latter was binned at the same radii where its kinemetry counterpart is defined, and a smoothing was applied in order to avoid spurious fluctuations, specially in the inner part where the number of pixels per bin is small. To determine the maximum stars contribution we followed a modified version of the method described in Simon et al. (2005), looking for the values of $\gamma_{\text {disk }}$ and $\gamma_{\text {bulge }}$ that minimize the $\chi_{v}^{2}$ without overpassing any point of the rotation curve. The very first point of each rotation curve was excluded because it may strongly affect the results and its uncertainty is large.

\section{Results}

\subsection{Zero Stars}

In table 2 we present the results of the zero disk test using the isothermal sphere model for the DM halo. We see that parameters $\rho_{0}$ and $R_{0}$ were similar for each galaxy for both RCs, but never remain the same. In order to check whether or not this change is statistically significant we should compute $\chi_{v}^{2}$ in a grid of values around this best solution, but this analysis is still in progress. Same comments apply to the case of NFW profiles whose results are not shown.

\begin{tabular}{ccccccc}
\hline \hline \multicolumn{7}{c}{ Zero Stars ISO } \\
\hline \hline & $\rho_{0}$ Pure Rotation' & $R_{0}$ & $\chi_{v}^{2}$ & $\rho_{0}$ & $R_{0}$ & $\chi_{v}^{2}$ \\
& $10^{-3} M \odot / p c^{3}$ & $k p c$ & & $10^{-3} M \odot / p c^{3}$ & $k p c$ & \\
\hline \hline ugc3876 & 202 & 1.9 & 3.05 & 182 & 1.57 & 1.83 \\
\hline ugc5842 & 92 & 2.9 & 4.22 & 63.7 & 2.5 & 0.74 \\
\hline ugc7045 & 422 & 1.9 & 3.13 & 570 & 1.41 & 7.56 \\
\hline ugc7876 & 159 & 1.9 & 2.23 & 107 & 1.7 & 2.36 \\
\hline ugc8334 & 1764 & 1.3 & 4.93 & 1808 & 1.12 & 44.7 \\
\hline ugc9866 & 924 & 0.96 & 1.56 & 854.4 & 0.72 & 1.21 \\
\hline ugc11300 & 229 & 1.7 & 3.56 & 247.2 & 1.38 & 5.15 \\
\hline \hline
\end{tabular}

Table 2: Comparison of best isothermal halo parameters obtained for each galaxy using both rotation curves.

More interesting results are those shown in table 3. It compares the quality of the fit for the ISO and NFW model. Those values of $\chi_{v}^{2}$ highlighted in green indicate the best model for each galaxy and rotation curve. If no model is highlighted in green, it means that both of them are equally good. In the case of the Kinemetry RC there is a favorite model for each galaxy, and one would conclude that there are 4 cuspy haloes and 3 flat haloes in our sample. On the other hand, results obtained using the "pure rotation" velocity cloud hardly allowed us to select a best option between the models. Only in two cases the NFW DM halo fits the RC much better than the Isothermal sphere, while the opposite never happens.

\subsection{Maximal disk}

Concerning the Maximal disk test, we present in table 4 the maximum values of $\gamma_{\text {bulge }}$ and $\gamma_{\text {disk }}$ allowed by each rotation curve. As can be seen, this time there is a big difference between the 


\begin{tabular}{ccccc}
\hline \hline \multicolumn{3}{c}{$\chi_{v}^{2}$ Zero Stars - Best Model } \\
\hline \hline \multicolumn{3}{c}{ Pure Rotation } & \multicolumn{2}{c}{ Kinemetry } \\
\hline ISO & NFW & ISO & NFW \\
\hline ugc3876 & 3.05 & 2.99 & 1.83 & 0.8 \\
\hline ugc5842 & 4.22 & 4.32 & 0.74 & 11.85 \\
\hline ugc7045 & 3.13 & 2.64 & 7.56 & 2.26 \\
\hline ugc7876 & 2.23 & 2.32 & 2.36 & 7.05 \\
\hline ugc8334 & 4.93 & 3.2 & 44.7 & 4.75 \\
\hline ugc9866 & 1.56 & 1.52 & 1.21 & 3.24 \\
\hline ugc11300 & 3.56 & 3.31 & 5.15 & 0.85 \\
\hline \hline
\end{tabular}

Table 3: Zero Stars - Best Model.

values obtained using kinemetry or "pure rotation". For instance, in the case of ugc7045 the "pure rotation" case allows the existence of a massive bulge in the center while kinemetry produces a very light central component, and even a smaller $\gamma_{\text {disk }}$ which means a much smaller overall baryonic mass content. As a general trend we notice that all $\gamma_{\text {disk }}$ are higher in the "pure rotation" case than in the kinemetry one, ugc8334 showing the biggest difference between them. Maximum bulges allowed tend to be more massive when one uses kinemetry, but the differences are less strong in general, so it seems that an overall higher baryonic content is allowed for the "pure rotation" RC. This fact is not really unexpected, given that the "pure rotation" RC tends to reach higher velocities than the kinemetry, by definition.

\begin{tabular}{ccccc}
\hline \hline \multicolumn{5}{c}{ Maximal stars comparison } \\
\hline \hline & 'Pure Rotation' & \multicolumn{2}{c}{ 'Kinemetry' } \\
\hline & $\gamma_{\text {disk }}$ & $\gamma_{\text {bulge }}$ & $\gamma_{\text {disk }}$ & $\gamma_{\text {bulge }}$ \\
\hline \hline ugc3876 & $7.19 \pm 0.67$ & $0.00+1.60$ & $5.31 \pm 0.82$ & $0.32 \pm 1.06$ \\
\hline ugc5842 & $1.86 \pm 0.61$ & $0.00 \pm 0.00$ & $0.74 \pm 0.30$ & $0.00 \pm 0.00$ \\
\hline ugc7045 & $3.32 \pm 0.01$ & $4.45 \pm 0.88$ & $2.93 \pm 0.50$ & $0.23 \pm 0.39$ \\
\hline ugc7876 & $1.81 \pm 0.83$ & $0.00 \pm 0.00$ & $0.41 \pm 0.56$ & $0.00 \pm 0.00$ \\
\hline ugc8334 & $2.55 \pm 0.18$ & $0.18 \pm 0.15$ & $0.80 \pm 0.13$ & $1.54 \pm 0.29$ \\
\hline ugc9866 & $3.29 \pm 0.06$ & $0.06 \pm 0.28$ & $2.14 \pm 0.47$ & $0.00+0.50$ \\
\hline ugc11300 & $2.01 \pm 0.34$ & $0.17 \pm 0.36$ & $1.98 \pm 0.12$ & $0.52 \pm 0.41$ \\
\hline \hline
\end{tabular}

Table 4: Comparison of mass-to-light ratios obtained when a maximal contribution from stars is allowed, in the "pure rotation" and kinemetry cases. All the values are expressed in $M \odot / L \odot$

\section{Summary and Conclusions}

Mass models of galaxies combining photometry and kinematics is currently the main way of probing galactic dark matter haloes. Here we present simplified mass models for a sample of seven galaxies previously studied in the context of the cusp/core problem, using their high-resolution velocity maps in two different ways. Our tests suggest that assuming pure rotation or effectively correcting for non-circular motions strongly affect the conclusions about the cuspiness of the DM halo and about the maximum baryonic content allowed by the data. Thus it is very important to study in more detail at which extent it is possible to recover true information about the shape of DM haloes from velocity fields, and what is the best way of using the data for this purpose. 


\section{Acknowledgments}

JCBP and CMdO would like to thank FAPESP for support (PhD fellowship 2011/21678-0 and thematic grant 2006/56213-9). We also want to acknowledge the VIII International Workshop on the Dark Side of the Universe organizers for financial support which allowed JCBP to participate in this conference.

\section{References}

[1] E. Athanassoula, A. Bosma, \& S. Papaioannou, Halo parameters of spiral galaxies, A\&A, 1987, 179, 23.

[2] K. G. Begeman, A. H. Broeils, \& R.H. Sanders, Extended rotation curves of spiral galaxies - Dark haloes and modified dynamics, MNRAS, 1991, 249, 523.

[3] W. J. G. de Blok, F. Walter, E. Brinks, C. Trachternach, S.H. Oh, \& R. C. Kennicutt, High-resolution rotation curves and galaxy mass models from THINGS, AJ, 2008, 136, 2648.

[4] W. J. G. de Blok, The Core-Cusp Problem, Advances in Astronomy, 2010.

[5] A. del Popolo, Statistical properties of the dark matter haloes of dwarf galaxies and correlations with the environment, MNRAS, 2012, 419, 971.

[6] J. Dubinski, \& R. G. Carlberg, The structure of cold dark matter halos ApJ, 1991, 378, 496.

[7] B. Epinat, P. Amram, M. Marcelin, C. Balkowski, O. Daigle, O. Hernandez, L. Chemin, C. Carignan,

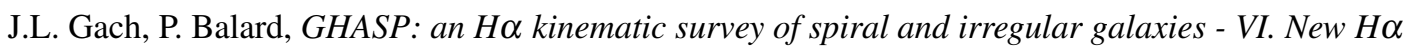
data cubes for 108 galaxies, MNRAS, 2008, 388, 500.

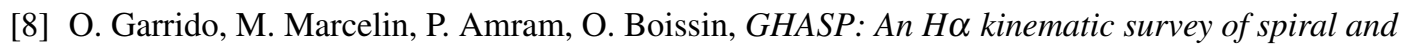
irregular galaxies. II. Velocity fields and rotation curves of 15 galaxies, A\&A, 2003, 399, 51.

[9] F. Governato, A. Zolotov, A. Pontzen, C. Christensen, S.H. Oh, A. M. Brooks, T. Quinn, S. Shen, J. Wadsley, Cuspy no more: how outflows affect the central dark matter and baryon distribution in $\Lambda$ cold dark matter galaxies, MNRAS, 2012, 422, 1231.

[10] D. Krajnović, M. Cappellari, P.T. de Zeeuw, Y. Copin, Kinemetry: a generalization of photometry to the higher moments of the line-of-sight velocity distribution, MNRAS, 2006, 366, 787.

[11] R. Kuzio de Naray, S. S. McGaugh, \& J. C. Mihos, Constraining the NFW potential with observations and modeling of low surface brightness galaxy velocity fields, ApJ, 2009, 692, 1321.

[12] J. F. Navarro, V. R. Eke, \& C. S. Frenk, The cores of dwarf galaxy haloes, MNRAS, 1996a, 283, L72.

[13] J. F. Navarro, C. S. Frenk, \& S. D. M. White, The Structure of Cold Dark Matter Halos, ApJ, 1996b, $462,563$.

[14] J. D. Simon, A. D. Bolatto, A. Leroy, L. Blitz, \& E. L. Gates, High-resolution measurements of the halos of four dark matter-dominated galaxies: deviations from a universal density profile, ApJ, 2005, $621,757$.

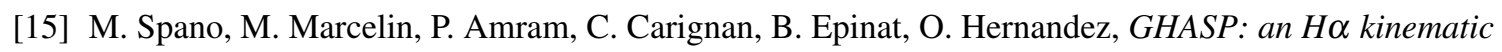
survey of spiral and irregular galaxies - V. Dark matter distribution in 36 nearby spiral galaxies, MNRAS, 2008, 383, 297. 